\title{
A Comparative Study of Technology-Mediated Learning Strategies for Teaching People with Intellectual Disability
}

\author{
Rodrigo Laiola Guimarães ${ }^{1}$ \\ UFES \\ Vitória, Brasil \\ rlaiola@inf.ufes.br
}

\author{
Andrea Britto Mattos \\ IBM Research \\ São Paulo, Brasil \\ abritto@br.ibm.com
}

\author{
Carlos Henrique Cardonha \\ IBM Research \\ São Paulo, Brasil \\ chcardo@br.ibm.com
}

\begin{abstract}
In this paper, we report on our experiences investigating the role of digital technology in face-to-face instruction of students with intellectual disability. In this process, we used a multi-method approach and our findings integrate results from focus groups, interviews, observations, iterative prototyping, and user evaluation. Ultimately, we hope that our work can motivate future research efforts and bring to light opportunities to be considered in the development of mobile-based education solutions.
\end{abstract}

\section{Author Keywords}

Educational technology; Self-paced instruction; Instructorled training; Face-to-face learning; Intellectual disability.

\section{ACM Classification Keywords}

K.3.1. Computers and Education: Computer Uses in Education - Computer-assisted instruction (CAI), Computer-managed instruction (CMI).

\section{INTRODUÇÃO}

Em sala de aula, professores constantemente lidam com alunos com diferentes habilidades, bem como estilos e ritmos individuais de aprendizagem. Além disso, alunos muitas vezes têm maneiras diferentes de organizar o tempo, seguir instruções e concluir suas tarefas. Esses aspectos tornam a condução de atividades de ensino desafiadoras [11], e o problema é ampliado em cenários envolvendo estudantes com diferentes níveis de deficiência intelectual (DI). Nesse contexto, a velocidade na qual uma atividade de ensino é conduzida pode desempenhar um papel importante tanto para aumentar o nível de atenção e aprendizagem de estudantes, quanto para diminuir comportamento disruptivo em sala de aula [12].

Nas últimas décadas, houve avanços significativos em termos de tecnologia assistiva [10], apoio à aprendizagem [5] e avaliação integrada para alunos com deficiência [1]. Além disso, trabalhos de pesquisa investigaram as implicações de práticas de controle [9] e apoio à autonomia em sala de aula em termos de comportamento e

Permission to make digital or hard copies of all or part of this work for personal or classroom use is granted without fee provided that copies are not made or distributed for profit or commercial advantage and that copies bear this notice and the full citation on the first page. To copy otherwise, or republish, to post on servers or to redistribute to lists, requires prior specific permission and/or a fee. IHC'17, Brazilian Symposium on Human Factors in Computing Systems. October 23-27, 2017, Joinville, SC, Brazil. Copyright 2017 SBC. ISBN XXX-XX-XXXX-XXX-X (pendrive) desenvolvimento de alunos [12]. Reconhecemos esses esforços e os utilizamos para direcionar nossa própria pesquisa; no entanto, neste trabalho, nos concentramos no estudo de apoios tecnológicos para ajudar professores a gerenciar o ritmo de ensino em sala de aula.

Nossa pesquisa baseia-se em um trabalho conjunto com uma organização não-governamental que presta assistência e treinamento a indivíduos com DI na cidade de São Paulo. Em uma parceria de longo prazo para investigar o impacto da utilização de apoios tecnológicos como forma de complementar o processo de aprendizagem dos alunos [2], utilizamos uma abordagem multi-método e integramos resultados de grupos focais, entrevistas com especialistas, observações de uma sessão educacional tradicional, e a avaliação de um Curso Online Aberto e Massivo (do inglês Massive Open Online Course ou MOOC) [6].

Nesse processo, identificamos uma série de requisitos para a aprendizagem de estudantes com DI [7]. Em particular, devido ao déficit de atenção e dificuldade em seguir instruções, observamos que constantemente o professor precisa trabalhar a disciplina e atenção dos alunos, para trazê-los de volta para o foco da atividade sempre que esses interagem com dispositivos móveis com tela sensível ao toque (no caso, tablets) em sala de aula. Portanto, no contexto de estratégias metodológicas de ensino para pessoas com DI, este trabalho investiga se professores podem se beneficiar de apoios tecnológicos para melhor gerenciar o ritmo de ensino e, consequentemente, reduzir o comportamento disruptivo de alunos em sala de aula.

\section{SOLUÇÃO/ABORDAGEM ADOTADA}

Com auxílio da organização parceira, recrutamos 11 pessoas para um estudo em suas instalações: 1 professor (do sexo masculino) com ampla experiência de ensino para pessoas com DI e 10 estudantes (3 mulheres), todos com grau moderado de DI. Os alunos tinham diferentes níveis de familiaridade com tablets, e podem ser considerados analfabetos funcionais (podem ler e, possivelmente, escrever frases simples com um vocabulário limitado). Os participantes não receberam compensação monetária e obtivemos o consentimento de todos eles (ou de seus representantes) para que participassem desse estudo.

\footnotetext{
${ }^{1} \mathrm{O}$ trabalho foi realizado quando o autor estava vinculado à IBM Research, São Paulo, Brasil.
} 
Os alunos foram divididos em 3 grupos (de 3, 3 e 4 pessoas) e cada participante (incluindo o professor) recebeu um tablet no início da sessão. Essencialmente, os dispositivos tinham acesso à rede local e a um aplicativo de e-learning que desenvolvemos para esse estudo ${ }^{2}$. Esse aplicativo suporta 2 estratégias de aprendizagem: uma autodirigida e outra guiada pelo professor. No primeiro caso (autodirigido), os alunos podiam navegar por conta própria por uma sequência de objetos de aprendizagem predefinidos pelo professor. Já a segunda estratégia (guiada), contava com o suporte de sincronização, isso é, o professor através de seu tablet, especificava o objeto de aprendizagem em que os alunos deveriam trabalhar em um dado momento e os seus respectivos dispositivos móveis eram automaticamente sincronizados de acordo.

\section{OBSERVAÇÕES E ANÁLISE DOS RESULTADOS}

As atividades em sala de aula, discussões em grupo ao término de cada sessão e entrevista individual com o professor nos possibilitou obter gravações audiovisuais, fotos e várias anotações manuscritas. Analisamos esses dados e usamos técnicas de codificação aberta [4] para identificar iterativamente os principais pontos que emergiram nas sessões. Nossas observações e quaisquer elementos demográficos são apresentados neste trabalho de forma agregada e não identificados a nível individual.

Nas sessões com suporte de sincronização, observamos que os alunos mais proficientes em matemática frequentemente ficavam entediados quando tinham que esperar pelos outros para poder avançar para o próximo exercício [8]. Assim que esses alunos perceberam que o ritmo da atividade era, de certa forma, ditado pela velocidade dos mais lentos, eles involuntariamente acabavam criando uma pressão sobre aqueles. Segundo o professor, ter a opção de bloquear a tela de um aluno pode ser útil, particularmente quando ele quiser que esse aguarde por instruções. Porém, deixar todos os alunos esperando pode ser um impedimento para o desenvolvimento da autonomia, além de poder constranger os alunos que têm mais dificuldades. De acordo com relatos do professor, um meio termo seria ter a possibilidade de permitir que os alunos avançassem até um certo ponto.

Já nas sessões sem suporte de sincronização, o principal desafio do professor foi ter que se dividir entre a coordenação do ritmo de ensino e a verificação visual se os alunos com DI estavam tendo dificuldades. Essa estratégia se mostrou ineficiente mesmo para um grupo pequeno. Provavelmente esse problema poderia ter sido minimizado se um apoio tecnológico baseado no uso de notificações para avisar quando um aluno terminava de responder um exercício tivesse sido disponibilizado para o professor. Durante a entrevista o professor disse ainda que essas notificações são bastante úteis e podem ajuda-lo a tomar uma ação imediatamente. Porém, na nossa implementação

\footnotetext{
${ }^{2}$ https://youtu.be/-XL6PoeMsOs
}

essas notificações não ficavam visíveis por muito tempo e a recomendação dele foi para que esse tempo fosse maior.

\section{Significância Estatística}

Com base nos registros de interação dos participantes, os quais capturamos através da instrumentação do aplicativo de e-learning, formulamos e testamos estatisticamente algumas hipóteses para verificar se havia correspondência entre os resultados qualitativos e quantitativos. Nesse processo, utilizamos extensivamente alguns pacotes estatísticos especializados, como IBM SPSS Statistics ${ }^{3}$ e $\mathrm{G}^{*}$ Power ${ }^{4}$. Para análise dos dados usamos a Análise de Variância (ANOVA). Alternativamente, poderíamos ter utilizado o teste $\mathrm{t}$, o qual é voltado para casos com apenas 2 grupos diferentes para a variável independente; porém, optamos pela ANOVA pois algumas variáveis independentes possuíam 3 ou mais grupos (por ex., faixa etária e número da sessão).

Resumidamente, a ANOVA é útil para determinar se há diferenças significativas nas médias de uma variável dependente medida em um nível contínuo (por exemplo, tempo e aproveitamento) em função de uma variável independente (chamada de fator) com 2 ou mais grupos distintos (categorias ou níveis). No nosso caso, as variáveis dependentes (ou seja, a quantidade de tempo que os alunos levaram para responder a um exercício e seu desempenho) e as variáveis independentes (por exemplo, estratégia de estimulação de instrução, gênero, idade, tipo de exercício etc.) satisfazia essas condições.

Vale ressaltar que os pré-requisitos para o uso da ANOVA são: as variáveis dependentes devem ser contínuas, as observações devem ser independentes e sem valores aberrantes (outlier) significativos, deve se ter homogeneidade de variância, a variável independente tem dois ou mais grupos independentes e a variável dependente deve ser aproximadamente normalmente distribuída para cada nível da variável independente. Quando essas premissas foram violadas e, consequentemente, o uso da ANOVA era inadequado, utilizamos o teste de KruskalWallis $\mathrm{H}$, que é considerado o equivalente não paramétrico.

Já para o teste de Kruskal-Wallis, as medidas precisam ser independentes umas das outras, a variável independente deve consistir em duas ou mais categorias, as variáveis dependentes devem ser medidas no nível discreto ou contínuo, o tamanho da amostra deve ser aproximadamente igual para cada possível valor assumido pela variável independente, a distribuição não precisa ser normal e as variâncias não precisam ser iguais. No entanto, para que a interpretação dos resultados do teste de Kruskal-Wallis $\mathrm{H}$ estejam corretas, é preciso ainda que as distribuições em cada grupo da variável independente tenham a mesma forma. Avaliamos essa premissa adicional através de

\footnotetext{
${ }^{3} \mathrm{http}: / / w w w . i b m . c o m / a n a l y t i c s / u s / e n / t e c h n o l o g y / s p s s /$

${ }^{4}$ http://www.gpower.hhu.de
} 
inspeção visual dos histogramas de distribuição de frequências da variável dependente para cada valor assumido pela variável independente.

\section{O Fator Estratégia de Aprendizagem}

$H_{0}$ : A estratégia de aprendizagem adotada nas sessões não teve efeito significativo sobre a quantidade de tempo que os alunos com DI levaram para responder um exercício $\left(\mu_{\text {tempo c/ sinc. }}=\mu_{\text {tempo s/sinc. }}\right)$.

$\mathrm{Na}$ avaliação da hipótese nula $H_{0}$ de que não houve diferença significativa na quantidade de tempo que os alunos com DI levaram para responder um exercício baseado na estratégia de aprendizagem adotada pelo professor, a variável independente, estratégia de aprendizagem, incluía dois grupos: sessões com $\left(\mu_{\text {tempo c sinc }}\right.$ $=22,28 \mathrm{~s}, \mathrm{SD}=14,27 \mathrm{~s}, \mathrm{n}=50)$ e sem $\left(\mu_{\text {tempo s} / \text { sinc. }}=49,69 \mathrm{~s}\right.$, $\mathrm{SD}=30,39 \mathrm{~s}, \mathrm{n}=45)$ suporte de sincronização.

Lançamos mão do G*Power para estimar se a ANOVA poderia ser usada para detectar um efeito com um determinado grau de confiança sobre nossa amostra. Nesse cálculo, utilizamos o tamanho do efeito $=0,96$ (calculado com base nos desvios médios e padrão), $\mathrm{p}=0,05$ (nível de significância $\alpha$ ou erro Tipo $\mathrm{I}^{5}$ ) e potência $=0,95$, e encontramos que uma amostra de 18 medidas seria suficiente $(<\mathrm{N}=95)$. Também realizamos uma análise da potência estatística no SPSS para determinar a probabilidade de cometer um erro de Tipo II (denotado por $\beta[3]$ ) e descobrimos que a potência observada era $\approx 1,00$ $(\mathrm{N}=95, \mathrm{p}=0,00$ e tamanho do efeito $=0,26)$. Isso significa que a probabilidade de ter um falso negativo (ou seja, quando falhamos em rejeitar $H_{0}$ e $H_{0}$ é realmente falsa) é $\approx$ $0 \%$ (potência $=1-\beta)$.

A maioria dos pré-requisitos para utilizarmos a ANOVA foi satisfeita. Para avaliar a condição de normalidade da variável dependente, utilizamos o teste de Shapiro-Wilk (estatística paramétrica). A hipótese nula neste caso foi que a quantidade de tempo que os alunos com DI levam para responder a um exercício não é estatisticamente significativamente diferente da distribuição normal. Ao executar o teste de Shapiro-Wilk em nossos dados, encontramos $\mathrm{p}=0,00$ para cada nível $(<0,05$ nível de significância $\alpha$ ). Portanto, rejeitamos a hipótese nula e presumimos que houve diferença estatisticamente significativa entre a variável dependente e a distribuição normal, de modo que a variável dependente não era normalmente distribuída. Nós também administramos o teste de normalidade de Kolmogorov-Smirnov para verificar novamente e tirámos as mesmas conclusões de que

\footnotetext{
${ }^{5}$ Nível de significância para a estatística: uma determinação é feita para rejeitar $(\mathrm{p}<\alpha)$ ou manter $(\mathrm{p}>\alpha)$ a hipótese nula. Um valor de $\mathrm{p}$ de 1 significa que aceitamos a hipótese nula como verdadeira; já um valor de $\mathrm{p}$ de 0 significa que aceitamos a hipótese nula como falsa.
}

os dados não eram normalmente distribuídos ( $\mathrm{p}$ novamente menor que o nível de significância $\alpha$ ).

Nesse caso, utilizamos o teste de Kruskal-Wallis H depois de verificar que todos os pré-requisitos eram satisfeitos para todos os grupos (inclusive a condição de que os histogramas de distribuição de frequências da variável dependente em cada grupo da variável independente tinham formas similares). Em seguida, executando o teste KruskalWallis $\mathrm{H}$ em nossos dados no SPSS, encontramos $\mathrm{p}=0,00$ $(<0,05$ nível de significância $\alpha)$, indicando que houve uma diferença estatisticamente significante na quantidade de tempo que os alunos com identificação levaram para responder a um exercício, dependendo da estratégia que o professor adotou em uma sessão de aprendizagem. Assim, rejeitamos a hipótese nula $H_{0}$ de que não houve diferença significativa na quantidade de tempo que os alunos com DI levaram para responder a uma pergunta com base na

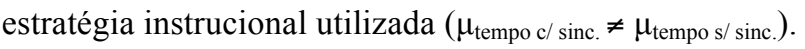

$H_{0}$ : A estratégia de aprendizagem adotada em uma sessão não teve efeito significativo no desempenho dos alunos com DI ( $\left.\mu_{\text {desempenho } \mathrm{c} / \text { sinc. }}=\mu_{\text {desempenho s/ sinc. }}\right)$.

Seguimos uma abordagem semelhante para avaliar a hipótese nula $H_{0}$ de que a estratégia de aprendizagem que o professor adotou durante uma sessão não teve efeito significativo no desempenho dos alunos em exercícios de múltipla escolha. Nas sessões nas quais foi utilizado o suporte de sincronização, obtivemos um desempenho médio $\mu_{\text {desempenho c/ sinc. }}=0.82(\mathrm{SD}=0,39, \mathrm{n}=50)$. Já nas outras sessões onde o suporte de sincronização não foi utilizado, obtivemos um desempenho médio $\mu_{\text {desempenho } \mathrm{s} / \text { sinc. }}=0,76$ $(\mathrm{SD}=0,44, \mathrm{n}=45)$.

Usamos o $\mathrm{G}^{*}$ Power para estimar se o tamanho de nossa amostra poderia ser usada para detectar um efeito com um determinado grau de confiança. Nesse cálculo, utilizamos o tamanho do efeito $=0,08$ (calculado com base em médias e desvios padrão), $\mathrm{p}=0,05$ e potência $=0,95$, e descobrimos que precisávamos de um total de 2182 amostras para obter um resultado estatisticamente significante. Infelizmente, em nosso estudo, não conseguimos obter um conjunto de dados tão grande devido a restrições de duração do experimento e número de participantes. $\mathrm{Na}$ análise de potência estatística usando o SPSS, encontramos $\mathrm{p}=0,45(>0,05$ nível de significância $\alpha$ ), o que também significa que, com base em nossa amostra $(\mathrm{N}=95$, tamanho do efeito $=0,006)$, o desempenho dos alunos com DI nas sessões de aprendizagem com suporte de sincronização não diferiu de forma estatisticamente significante do desempenho dos alunos em sessões de aprendizagem sem esse suporte. Portanto, neste caso, não pudemos rejeitar a hipótese nula $H_{0}$ de que não houve diferença significativa no desempenho de alunos com DI com base na estratégia de aprendizagem que o professor usa. 


\section{Outros Fatores}

Seguimos o mesmo procedimento descrito na seção anterior para analisar o efeito de outras variáveis independentes como gênero, faixa etária e número da sessão - na quantidade de tempo que os alunos com DI levaram para responder e seu desempenho em um exercício. Utilizando o $\mathrm{G}^{*}$ Power descobrimos que para todos esses fatores, precisaríamos de uma amostra muito maior $(>\mathrm{N}=95)$ para tirar conclusões estatisticamente significativas $(\mathrm{p}=0,05 \mathrm{e}$ potência $=0,95)$. Nós também realizamos uma análise de potência estatística no SPSS e confirmamos que, com base no tamanho da nossa amostra, não houve diferença estatisticamente significante no desempenho e na quantidade de tempo que os alunos com DI levaram para responder a um exercício com base nos fatores gênero, faixa etária e número de grupo $(\mathrm{p}>0,05$ nível de significância $\alpha$ ). Assim, não rejeitamos nenhuma hipótese nula de que não houve diferença significativa no desempenho ou na quantidade de tempo que os alunos com DI levaram para responder a um exercício com base nos diferentes valores que essas variáveis podem assumir.

Quanto ao fator tipo de exercício, constatamos que houve uma diferença estatisticamente significativa tanto no desempenho quando na quantidade de tempo que os alunos levaram para responder os exercícios de adição em relação aos de subtração; porém, nossa análise concluiu que não há efeitos de interação estatisticamente significativos entre os fatores estratégia de aprendizagem e tipo de exercício. Em outras palavras, isso significa que o efeito de cada variável independente é completamente separado da outra variável independente e, portanto, devemos concentrar-nos apenas nos principais efeitos anteriormente discutidos.

\section{CONCLUSÃO}

Neste trabalho, apresentamos uma breve visão de nossas experiências na indústria investigando o uso de apoios tecnológicos no ensino de estudantes com DI. Nesse processo, lançamos mão de métodos qualitativos e quantitativos de Interação Humano-Computador (IHC). Vale destacar que a principal contribuição de nosso trabalho não está na adoção de uma estratégia metodológica, de uma plataforma educacional, de um dispositivo ou mesmo de um suporte tecnológico específico, mas sim no entendimento do papel da tecnologia e na elicitação de oportunidades para iniciativas educacionais direcionadas a pessoas com DI.

Nossos resultados sugerem que o suporte de sincronização ajudou o professor a gerenciar melhor o ritmo de ensino da atividade de aprendizagem mediada pela tecnologia. No entanto, essa abordagem nem sempre é a melhor para estudantes com DI. Nossos resultados indicam ainda que a abordagem autodirigida pode ajudar a desenvolver a autonomia (por exemplo, permitir que os alunos com DI tomem a iniciativa) e dar aos professores mais flexibilidade para atender necessidades particulares (por exemplo, dar suporte personalizado quando necessário). Dessa forma, sugerimos que trabalhos futuros investiguem a combinação entre a aprendizagem tradicional, guiada pelo professor, e a aprendizagem autodirigida, ambas com auxílio de apoio tecnológico para melhor se ajustar às necessidades de professores e alunos com DI.

\section{AGRADECIMENTOS}

Este trabalho foi parcialmente financiado pelo Ministério da Ciência e Tecnologia do sob o contrato de $n^{\circ}$. FINEP 03.11.0371.00. Agradecemos os participantes da instituição parceira que possibilitaram a realização desse estudo.

\section{REFERENCES}

1. Julia Allen. 2015. Evidence-based Practices in the Design of Interactive Multimedia for Learners with Cognitive Learning Disabilities. In Proc. SITE '15, 2944-2949.

2. Carlos H. Cardonha et al. Toward a platform to support vocational training of people with disabilities. IBM Journal of $R \& D, 59,6,2: 1-2: 7$, Nov.-Dec. 2015.

3. Jacob Cohen. 1962. The statistical power of abnormal social psychological research: A review. Journal of Abnormal and Social Psychology, 65, 145-153.

4. Juliet M. Corbin and Anselm Strauss. 1990. Grounded theory research: Procedures, canons, and evaluative criteria. Qualitative Sociology, 13, 1, 3-21.

5. Meg Cramer et al. 2011. Classroom-based assistive technology: collective use of interactive visual schedules by students with autism. In Proc. of CHI.

6. Rodrigo L. Guimarães and Andrea B. Mattos. 2015. Exploring the Use of Massive Open Online Courses for Teaching Students with Intellectual Disability. In Proc. ASSETS, 343-344.

7. Rodrigo L. Guimarães et al. 2014. Apoio Tecnológico para Treinamento Vocacional. Deficiência Intelectual, 4, 7 (Jul/Dez), 34-39. ISSN: 2238-4618.

8. Rodrigo L. Guimarães, Andrea B. Mattos, and Carlos H. Cardonha. 2016. Investigating Instructional Pacing Supports for Teaching Students with Intellectual Disability. In Proc. of CHI EA ‘ 16, 2171-2177.

9. Johnmarshall Reeve. 2009. Why Teachers Adopt a Controlling Motivating Style Toward Students and How They Can Become More Autonomy Supportive. Educational Psychologist, 44, 3, 159-175.

10. Joe Reichle. 2011. Evaluation Assistive Technology in the Education of Persons with Severe Disabilities. Journal of Behavioral Education, 20, 1, 77-85.

11. Steven M. Ross and Ernest A. Rakow. 1981. Learner control versus program control as adaptive strategies for selection of instructional support on math rules. Journal of Educational Psychology, 73, 5, 745-753.

12. Candice R. Stefanou et al. 2004. Supporting autonomy in the classroom: Ways teachers encourage student decision making and ownership. Educational Psychologist, 39, 2, 97-110. 